\title{
Gas-Phase Nucleation of Diamond Clusters upon a Sharp Rise in Temperature under Conditions of CVD Synthesis
}

\author{
N. I. Alekseev ${ }^{a, *}$, V. S. Khadutin ${ }^{a}$, and I. K. Khmel'nitskii ${ }^{a}$ \\ ${ }^{a}$ Leningrad Electrotechnical Institute, St. Petersburg Electrotechnical University, St. Petersburg, 193076 Russia \\ *e-mail: NIAlekseyev@yandex.ru \\ Received February 13, 2021; revised May 10, 2021; accepted May 11, 2021
}

\begin{abstract}
It is shown that the predominant nucleation of diamond nuclei (instead of graphite ones) in plasma or hot-filament assisted CVD technology is due to a sharp increase in the temperature of the region of the initial gas mixture's motion. The nucleation of diamond nuclei then occurs immediately in the gas phase. The reason for such predominant nucleation is high oversaturation by small hydrocarbon fragments in the gas mixture, due to a rapid change in temperature and substantial differences between the desorption of such fragments from the surfaces of nuclei and the oxidation of nuclei. A way is described of synthesizing massive diamonds without the use of high pressures and CVD technology in its traditional form.
\end{abstract}

Keywords: diamond synthesis, chemical vapor deposition, quantum-chemical modeling

DOI: $10.1134 / \mathrm{S} 0036024421120025$

\section{INTRODUCTION}

This work continues our study [1] on the gas-phase formation of carbon cluster nuclei under conditions that hypothetically reveals the predominant emergence and growth of diamond (instead of graphite) clusters. These include the rapid heating (on the order of $1000 \mathrm{~K} / \mathrm{s}$ ) of the gas-phase medium of a carbon support as it approaches the substrate, as is done in modifications of the CVD technology used in diamond synthesis. Such modifications include PECVD (plasma enhanced CVD), microwave assisted CVD (the abbreviation MWCVD is also used), and hot filament assisted CVD (hot filament CVD or HFCVD). The same condition is apparently observed in HPHT technology as well (i.e., the synthesis of diamond from a solid carbon support such as graphite at high pressures and temperatures $T$ [2].

Our aim was to determine the importance of this condition (which we call the rapid heating criterion [1]) in CVD from the viewpoint of new phase nucleation, and to formulate possible approaches to synthesizing massive diamonds without limitations on their HPHT, MWCVD, and HFCVD quality.

\section{EFFECT THE SLOWER DYNAMICS OF THE DESORPTION OF SMALL HYDROCARBON FRAGMENTS HAS ON THE DEGREE OF OVERSATURATION, RELATIVE TO AN INCREASE IN TEMPERATURE}

According to the model proposed in [1], the nucleation of clusters of graphite or diamond nuclei occurs upon oversaturation in its source, i.e., the vapor of small hydrocarbon fragments (methyl and ethyl radicals), the formation of which from the initial gas mixture (hydrogen-methane-oxygen) grows upon a rise in temperature more quickly than the concentration of the same fragments desorbed from the surfaces of clusters. This is generally because, in contrast to the synthesis of a vapor source, desorption is a much greater multistage reaction that does not keep up with an increase in temperature. The equation describing the dynamics of attaining equilibrium level $N_{1}^{\text {(source,eq) }}(T(t))$ via source concentration $N_{1}^{\text {(source) }}$ at an instantaneous current temperature $T(t)$ is

$$
\dot{y}_{1}+y_{1} \frac{d \ln N_{1}^{\text {(source,eq) }}}{d t}=w_{01} \frac{N}{N_{1}^{\text {(source,eq) }}}\left(1-y_{1}\right),
$$

where $y_{1}=N_{1}^{\text {(source) }} / N_{1}^{\text {(source,eq) }}(T(t))$ is the relative concentration; $w_{01}=(T / h)\left(\Omega_{\mathrm{btn}} / \Omega_{0}\right) \exp \left(-E_{\mathrm{btn}}^{(\mathrm{diss})} / T\right)$ is the effective probability of methane dissociation within the theory of a transitional state [3]; $N \gg N_{1}^{\text {(source,eq) }}$ is the overall concentration of carbon atoms in the initial methane and the source; $N_{1}^{\text {(source,eq) }}(T(t))=\left(\Omega_{1} / \Omega_{0}\right) \exp \left(-E_{1} / T\right)$, where $E_{1} \approx$ $1.6 \mathrm{eV}$ is the difference between the required energies of dissociation of the final and initial products formed during the synthesis of a methyl radical from methane and hydrogen per single $\mathrm{CH}_{3}$ radial; $E_{\mathrm{btn}}^{\text {(diss) }}>E_{1}$ is the activation barrier of the source's synthesis; $\Omega$ denotes the vibrational and rotational components of the sta- 
tistical sums of the initial product (methane), the transitional state, and the source of nucleation; and the upper dot is the symbol for time differentiation.

In Eq. (1), it is assumed for simplicity that the generation of methyl radicals is a single-stage process of methane dissociation, and activation energy $E_{\mathrm{btn}}^{(\mathrm{dis})}$ is its only bottleneck. If a radical appears as a result of interaction between methane and oxygen or water vapor that pulls one hydrogen atom out of a $\mathrm{CH}_{4}$ molecule, energy $E_{\mathrm{btn}}^{\text {(diss) }}$ is less than $6 \mathrm{eV}$, according to data in the HyperChem quantum-chemical software suite [1] we used.

It is not difficult to solve Eq. (1) exactly, but an analog of such a solution for the multistage reaction of desorption (which is the opposite of nucleation and is considered below) obtained by solving the equation in partial derivatives can only be done numerically. Our solution was therefore constructed via interpolation between limiting cases. With

$$
\dot{y}_{1} \ll y_{1}(d / d t)\left(\ln N_{1}^{(\text {source }, \mathrm{eq})}\right),
$$

which corresponds to comparatively high temperatures. Equation (1) has the solution

$$
\begin{aligned}
y_{1} & =\left(1+\frac{E_{1}}{T^{3}} h \dot{T} \frac{\Omega_{1}}{\Omega_{\mathrm{btn}}} \exp \left(\frac{E_{\mathrm{btn}}^{(\mathrm{diss})}-E_{1}}{T}\right)\right)^{-1} \\
& \approx 1-\frac{E_{1}}{T^{3}} h \dot{T} \frac{\Omega_{1}}{\Omega_{\mathrm{btn}}} \exp \left(\frac{E_{\mathrm{btn}}^{\text {(dis) }}-E_{1}}{T}\right)
\end{aligned}
$$

and in the opposite case,

$$
\begin{aligned}
y_{1} \approx 1 & -\exp \left(-\frac{T^{3}}{\left(E_{\mathrm{btn}}^{\text {(dis) }}-E_{1}\right) h \dot{T}} \frac{\Omega_{\mathrm{btn}}}{\Omega_{1}}\right. \\
& \left.\times \exp \left(-\frac{E_{\mathrm{btn}}^{\text {(diss) }}-E_{1}}{T}\right)\right) .
\end{aligned}
$$

Concentration $N_{1}^{\text {(source,eq) }}(T)$ is estimated according to the law of acting masses:

$$
\begin{aligned}
& N_{1}^{\text {(source,eq) }} \approx N_{\mathrm{H}_{2}}^{1 / 2} N_{\mathrm{CH}_{4}}\left(h^{2} / 2 \pi m_{\mathrm{H}_{2}} T\right)^{3 / 4} \\
& \quad \times\left(m_{\mathrm{CH}_{3}} / m_{\mathrm{CH}_{4}}\right)^{3 / 2} V^{\prime} \exp \left(-E_{1} / T\right),
\end{aligned}
$$

where $v^{\prime} \sim 1$ is the ratio of the statistical sums of participants on the right side of the equation to the statistical sums of participants on its left side. It is a weak function of $T$.

The process opposite to the nucleation of carbon clusters from a vapor source is the desorption of small fragments from them. The chain of reactions in this process was considered in [1], where it was shown that the effective activation barrier of the chain of reactions $q_{\mathrm{btn}}^{(\mathrm{des})}$ for diamond clusters considers the need for the dissociation of $\mathrm{H}_{2}$ molecules to atomic hydrogen (which fills the disrupted valences of surface carbon atoms), and $q_{\mathrm{btn}}^{(\mathrm{des})}$ is nearly $6 \mathrm{eV}$ in a channel of both diamond and graphite.

In such a multistage reaction of desorption, we must make strong simplifications to obtain a solution under quickly changing temperature conditions. The approximate expression describing the dynamics of a change in the concentration of desorbed fragments in the same case of Eq. (2) and the one derived in Appendix A have the form

$$
\begin{gathered}
N_{1}^{(\mathrm{des})} / N_{1}^{(\mathrm{des}, \mathrm{eq})} \approx \exp \left(-\frac{h \dot{T}}{T^{2}}\right. \\
\left.\times \int_{0}^{s_{\mathrm{btn}}} \frac{q_{\mathrm{s}}^{(\mathrm{des})}}{(d / d s)\left(q_{\mathrm{s}}^{\text {(des) }}+q_{\mathrm{a}, \mathrm{s}}^{(\mathrm{des})}\right)} \exp \left(\frac{q_{\mathrm{a}, \mathrm{s}}^{(\mathrm{des})}}{T}\right) d \mathrm{~s}\right),
\end{gathered}
$$

where $N_{1}^{(\mathrm{des}, \text { eq })}(T(t))$ is the equilibrium concentration of desorbed fragments, assuming there is a constant concentration of methane. Integration variable $s$ is the reaction's number of stages; $q_{\mathrm{a}, \mathrm{s}}^{(\mathrm{des})}$ is the activation energy of the $s$ th stage; and $q_{\mathrm{s}}^{(\mathrm{des})}$ is the overall energy of dissociation of products at the sth stage, counted from the level for the desorption of the initial reagents. If $q_{\mathrm{s}}^{(\mathrm{des})}$ depends linearly on $s$, and the activation energy is constant $\left(q_{\mathrm{a}, \mathrm{s}}^{(\mathrm{des})}=q_{\mathrm{a}}^{(\mathrm{des})}\right)$,

$$
N_{1}^{(\mathrm{des})} / N_{1}^{(\mathrm{des}, \mathrm{eq})} \approx \exp \left(-\frac{h \dot{T}}{T^{2}} \frac{s_{\mathrm{btn}}^{2}}{2} \exp \left(\frac{q_{\mathrm{a}}^{(\mathrm{des})}}{T}\right)\right) .
$$

A similar expression under the condition opposite Eq. (2) (low temperatures) is derived in Appendix A.

When estimating the equilibrium concentration of fragments $N_{1}^{\text {(des,eq) }}(T)$, we assume (as in the calculations in [1]) that desorption from a diamond or graphene $\mathrm{C}_{n} \mathrm{H}_{k}$ cluster occurs via the reaction $\mathrm{C}_{n} \mathrm{H}_{k}+2 \mathrm{H}_{2} \leftrightarrow \mathrm{C}_{n-2} \mathrm{H}_{k}+\mathrm{C}_{2} \mathrm{H}_{4}$, where $n$ and $k$ are the numbers of carbon and hydrogen atoms in a cluster. Since the concentrations of $\mathrm{C}_{n} \mathrm{H}_{k}$ and $\mathrm{C}_{n-2} \mathrm{H}_{k}$ clusters are similar, the law of acting masses yields

$$
N_{1}^{(\text {des,eq })} \approx N_{\mathrm{H}_{2}}^{2}\left(h^{2} / 2 \pi m_{\mathrm{H}_{2}} T\right)^{3 / 2} V \exp \left(-q_{1} / T\right),
$$

where $q_{1}=E_{\mathrm{C}_{2} \mathrm{H}_{4}}-2 E_{\mathrm{H}_{2}}+E_{\mathrm{C}_{n-2} \mathrm{H}_{k}}-E_{\mathrm{C}_{n} \mathrm{H}_{k}}$ is the change in the overall energy of dissociation of the participants during the desorption of ethylene (the dominant component of the small desorbed hydrocarbon fragments) and constant $v$ has the same meaning as $v^{\prime}$ in Eq. (3). 


\section{ESTIMATING THE MOMENT \\ OF NUCLEATION UNDER CONDITIONS OF FALLING OVERSATURATION IN A VAPOR OF SMALL HYDROCARBON CLUSTERS}

Equations (3)-(7) show that concentration $N_{1}^{(\mathrm{des})}$ falls exponentially as $\dot{T}$ rises. Oversaturation grows exponentially, but the dependence on $T$ (at the model level) is superexponential. Logarithm of oversaturation $\zeta=\ln S$, a component of value

$$
\begin{gathered}
\varsigma \approx\left[\ln \frac{N_{\mathrm{H}_{2}}}{N_{\mathrm{CH}_{4}}}+\frac{1}{T}\left(q_{1}-E_{1}\right)\right. \\
\left.+\frac{h \dot{T}}{T^{3}}\left(\frac{T s_{m}^{2}}{2} \exp \left(\frac{q_{\mathrm{a}}^{(\mathrm{des})}}{T}\right)-E_{1} \exp \left(\frac{E_{\mathrm{a}}-E_{1}}{T}\right)\right)\right]
\end{gathered}
$$

falls sharply as $T$ rises, and its negative derivative $(d \varsigma / d t)_{\text {nat }}$ associated with the natural change in oversaturation falls equally in absolute value.

Let us determine the moment when $(d \zeta / d T)_{\text {nat }}$ in the $\zeta(T)$ curve is lower in absolute value than rate of nucleation $(d \zeta / d T)_{\text {nucl }}$. It is known [4] that $(d \zeta / d T)_{\text {nucl }}$ must be described in different ways at high and relatively low oversaturations. Nucleation is almost barrierless in the first case, and is explosive with a rate proportional to $\exp \left(-\Delta G_{\mathrm{c}}^{(m)} / T\right)$ and critical barrier $G_{\mathrm{c}}^{(m)}$ to nucleus formation at a previously unknown maximum oversaturation in the second. To determine this oversaturation, we must solve the problem of explosive nucleation [5].

The concentration of saturated vapor over the surfaces of clusters in our case forms after a long delay, and condensation develops from ultimate oversaturations. We must therefore start with a barrierless case. For the rate of change in $\zeta$ during condensation, we write

$$
(d \varsigma / d t)_{\text {nucl }}=\dot{T}\left(\varsigma / N_{1}^{(\text {source })}\right)\left(d N_{1}^{(\text {source })} / d T\right),
$$

and estimate drop $d N_{1}^{\text {(source) }} / d t$ in the concentration of small fragments as a source of nucleation (subscript 1 denotes a conditional monomer) using the rate of dimerization:

$$
\begin{aligned}
& d N_{1}^{\text {(source) }} / d T=-\left(\sigma_{\mathrm{crs}} V_{\mathrm{T}} / \dot{T}\right) \\
& \times\left(N_{1}^{\text {(source) }}\right)^{2} \exp \left(-E_{\text {rep }} /(T)\right),
\end{aligned}
$$

where $\sigma_{\text {crs }}$ in the cross section of fragment collisions emphasizes the difference from the surface tension (which is also denoted as $\sigma$ ) and $E_{\text {rep }}$ is the energy of repulsion during the coalescence of fragments. Using Eq. (2), we can easily demonstrate that temperature $T_{\text {cross }}$ corresponding to the intersection between curves $\left|(d \varsigma / d T)_{\text {nat }}\right|$ and $\left|(d \varsigma / d T)_{\text {nucl }}\right|$ depends logarithmically

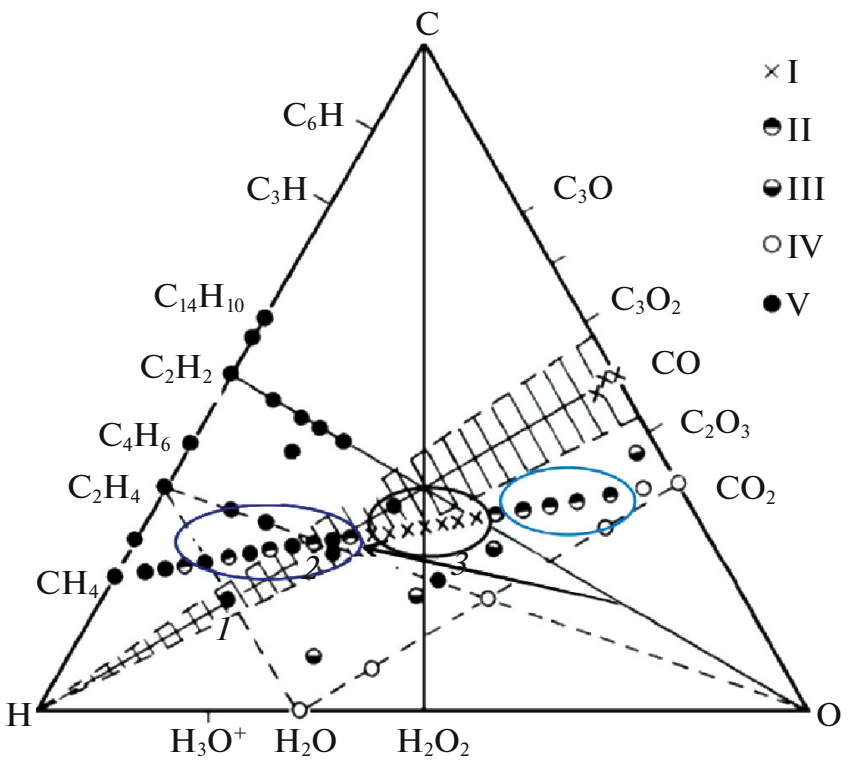

Fig. 1. Diagram of the conditions of stoichiometric diamond and graphite polycondensation with the conversion of diamond and graphite by oxidizers (the plot was taken from [7] and greatly simplified): I (the central region in the triangle with crosses) is the formation of diamond on the substrate due to the overgrowth of diamond material; II is diamond graphitization (when a $\mathrm{CH}_{4}+\mathrm{CO}_{2}$ mixture is used); III is the selective oxidation of graphite with the retention of diamond; IV is the complete oxidation of graphite and diamond; and $\mathrm{V}$ denotes organic materials (with no details).

on $q_{\mathrm{a}}^{\text {(des) }}$ and is $\sim 1500 \mathrm{~K}$ at $\dot{T}=1000 \mathrm{~K} / \mathrm{s}$ and concentration $N_{\mathrm{CH}_{4}}=100$ Torr. This means condensation cannot start until the temperature reaches a certain value.

At nearly equal values of parameter $q_{\mathrm{a}}^{(\mathrm{des})}$, nucleation can start in the channels of both graphite and diamond. If $q_{\mathrm{btn}}^{\text {(eff) }}$ of diamond is slightly higher than that of graphite, the conditions for condensation form earlier in the channel of graphite. If the nucleation of graphite clusters is impossible for one reason or another, however, the nucleation of diamond clusters is virtually instantaneous. One reason why it might be impossible could be the presence of oxygen. In MWCVD and HFCVD, it is the presence of oxygen that ensures a choice between the diamond and graphite channels, since the stabler diamond nuclei are oxidized much less than those of graphite, both in the volume and on the surface. This is clearly observed in the familiar Bachmann diagram (Fig. 1), showing that a certain amount of oxygen is absolutely necessary the synthesis of diamond.

It is therefore natural to think that the nucleation of carbon clusters must occur in the diamond channel. To make a better estimate, however, we must consider the possibility of explosive nucleation at any current $T$ 


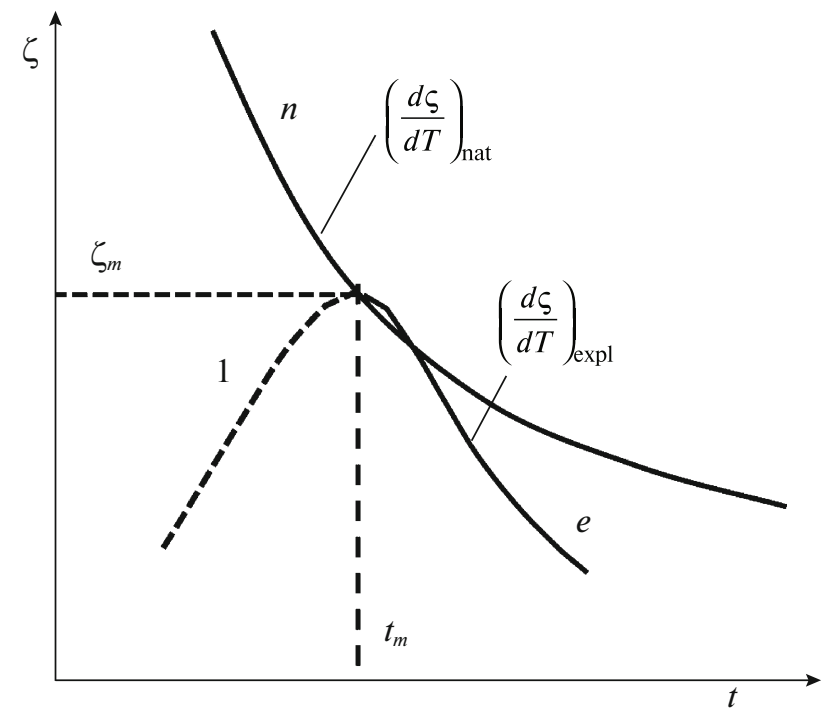

Fig. 2. Scheme for estimating the temperature of explosive nucleation upon a natural drop in oversaturation caused by a rise in temperature. Curves $n$ and $e$ characterize the drop in the logarithm of oversaturation $\zeta$, which is natural $(n)$ and explosive (induced explosive nucleation) (e). (1) Fictitious background of the emergence of a metastable state with the specified logarithm of oversaturation $\zeta_{m}$.

and oversaturaton $\zeta(T)$, assuming that these values correspond to a certain metastable state. The prehistory for the emergence of such a state could lie in Fig. 2, to the left of moment $t_{m}$. For our problem, it is minor and shown by dashed line 1 .

We must therefore solve the problem of the relaxation of a metastable state with parameters $\zeta_{m}, t_{m}$, and $T_{m}$, which are associated with current values $\zeta, t$, and $T$. The explosive stage of relaxation terminates when rate $\left|(d S / d T)_{\text {expl }}\right|$ of the drop in oversaturation reaches its maximum and starts to fall (the lower arrow in Fig. 2). In the semi-analytical solution to the classic nucleation problem [5-7], this rate is estimated by assuming there is a law of quadratic descent for $\zeta(T)$ after maximum $\zeta_{m}$. The rate of the drop in $\zeta$ can then be considered maximal when the drop in true source concentration $N_{1}$ relative to $N_{1}^{\text {(source) }}$ described by Eq. (1) is $1-1 / e$; i.e., $\Delta N_{1} / N_{1}^{\text {(source) }} \approx 1-1 / e$. If the explosive stage is too slow relative to $\left|(d \zeta / d T)_{\text {nat }}\right|$, condensation is impossible with this law of change in $\zeta(T)$. In contrast, if the half width of the maximum in the explosive condensation curve is small, condensation is determined by the moment when curves $\left|(d \zeta / d T)_{\text {nat }}(T)\right|$ and $\left|(d \zeta / d T)_{\text {expl }}(T)\right|$ intersect.

As in the classic nucleation problem [5-7], the process is calculated using the equation of balance between the concentration of the substance (carbon) in the carbon fragments and the products of their nucleation (e.g., diamond or graphite clusters). The drop in source concentration $N_{1}$ with respect to $N_{1}^{\text {(source) }}$ in Eq. (2) is equal to the amount of condensed fragments $\Delta N_{1}$ :

$$
N_{1}^{\text {(source) }}-N_{1}=\Delta N_{1}=\int_{0}^{t} J(\tau) g(\tau \rightarrow t) d \tau
$$

for small $\mathrm{CH}_{n}$ and $\mathrm{C}_{2} \mathrm{H}_{m}$ fragments, the coalescence and rearrangement of which form a cluster of nuclei. For simplicity, we assume that the concentration of the source of nucleation of diamond or graphite clusters changes slightly to describe flux $J\left[\mathrm{~cm}^{-3} \mathrm{~s}^{-1}\right]$ of clusters through the bottleneck in the space of sizes $\left(x_{\mathrm{c}}, \Delta G_{\mathrm{c}}\right)$ in Eq. (11) by a version of Zeldovich's formula. We use a quasi-steady state approach, assuming that the flux reflects the change in oversaturation immediately, and the density of the spatial flux of fragments to a growing nucleus is determined by Knudsen's expression $1 / 4 N_{1} v_{T}$ :

$$
\begin{gathered}
J^{-1}=\left(1 / N_{1}\right) \sum_{g=1}^{\infty}\left(w_{g} \Omega_{g}\right)^{-1} \\
\approx\left(\pi N_{1}^{2} V_{T}\right)^{-1}\left(4 \pi c / 3 g_{c}\right)^{2 / 3} \int_{1}^{\infty} d g / \Omega_{g} ; \\
J \approx \frac{N_{1}^{2} V_{T}}{6 S c}\left(\frac{4 \pi c}{3}\right)^{1 / 3} \exp \left(-\frac{\Delta G_{\mathrm{c}}}{T}\right) \sqrt{\frac{2 \pi T \Phi \sigma_{1}}{\left(\sigma_{1}-\varsigma T\right)^{2}}},
\end{gathered}
$$

where $c$ is the concentration of carbon atoms in the condensed carbon phase; $v_{T}$ is the thermal velocity of source particles; and the denominator is complemented by the Cortney factor (oversaturation $S$ ). Critical barrier to nucleation $\Delta G_{\mathrm{c}}$ was determined in [1] by minimizing Gibbs free energy $\Delta G_{g}$ of an ensemble of $g$ atoms when they form a cluster [1]:

$$
\begin{gathered}
\Delta G_{g}=\sigma_{1} g(1+\bar{\varpi} x)^{-1}-g_{\varsigma} T, \quad \text { where } \\
\bar{\sigma}=(36 \pi)^{-1 / 3} \approx 0.207, \quad x=g^{1 / 3} .
\end{gathered}
$$

The second multiplier in the subintegral expression of Eq. (11) is the rate of increase in the number of atoms $g$ in a cluster:

$$
\frac{d g}{d t}=4 \pi r_{g}^{2} c \frac{d r_{g}}{d t}=N_{1} v_{T} \pi r_{g}^{2},
$$

which shows radius $r_{g}$ of a cluster grows as

$$
\begin{gathered}
r_{g}=\frac{N_{1} V_{T} t}{4 c}+r_{c} \quad \text { and at } \quad r_{g} \gg r_{c} \\
g=\frac{4}{3} \pi c\left(\frac{N_{1} V_{T} t}{4 c}\right)^{3}=\frac{\pi N_{1}^{3} v_{T}^{3} t^{3}}{48 c^{2}} .
\end{gathered}
$$

Both subintegral multipliers in Eq. (12) are thus determined. Reducing Eq. (11) to the set of algebraic equations in [5-7] requires single and then double time differentiation, after which the system is closed by the equation for the equilibrium concentration of a vapor 


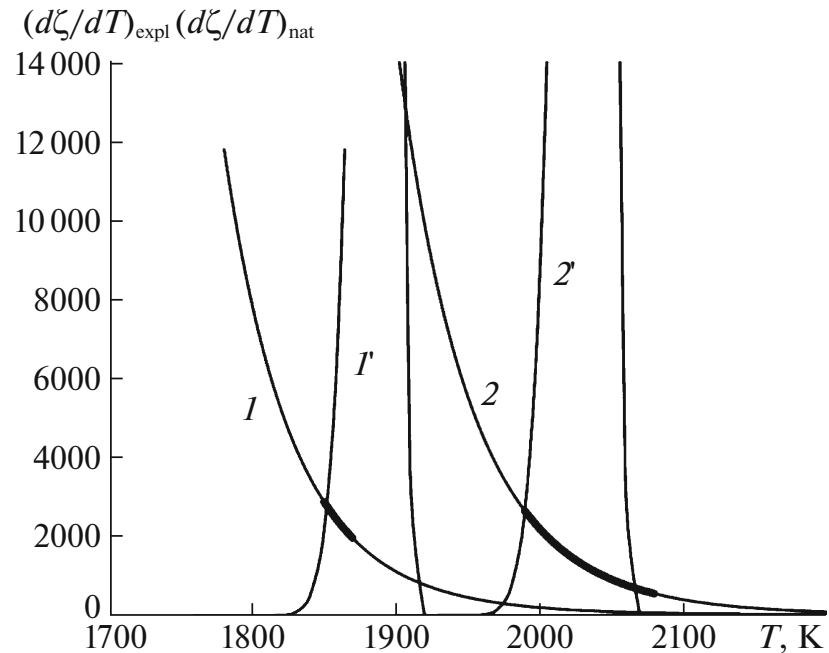

Fig. 3. Change in the rate of the drop in the logarithm of oversaturation in channels of $\left(1,1^{\prime}\right)$ graphite and $\left(2,2^{\prime}\right)$ diamond. $(1,2)$ Natural due to a rise in temperature; $\left(1^{\prime}, 2^{\prime}\right)$ explosive due to the explosive nucleation of clusters.

source. There is then no need for these procedures. The initial oversaturation and temperature are parameters, and the subintegral function in Eq. (12) can be written by separating the powers of $N_{1}$ and allowing for the difference between $N_{1}$ and $N_{1}$ (source) caused by the nucleation of clusters and a gas source transitioning into them by moment $t$ :

$$
\begin{gathered}
J(\tau) g(\tau, t)=\theta \sum_{p=0}^{5}\left[C_{5}^{p}\left(-\frac{E_{1}}{T^{3}} h \dot{T} \frac{\Omega_{1}}{\Omega_{\mathrm{btn}}}\right)^{p}\right. \\
\left.\times \exp \left(\left[p\left(E_{\mathrm{btn}}^{(\mathrm{diss})}-E_{1}\right)-\left(\Delta G_{\mathrm{c}}+5 E_{1}\right)\right] / T-\varsigma\right)\right] \\
\times(t-\tau)^{3},
\end{gathered}
$$

where $\Delta G_{\mathrm{c}}=\sigma_{1} x_{\mathrm{c}}{ }^{4} /(4 T) ; C_{5}^{p}$ is the factorial multiplier in the Newton binomial; and

$$
\begin{gathered}
\theta=\frac{\pi V_{T}{ }^{4}}{288 c^{3}}\left(\frac{4 \pi c}{3}\right)^{1 / 3} \sqrt{\frac{2 \pi T \varpi \sigma_{1}}{\left(\sigma_{1}-\varsigma T\right)^{2}}} \\
\times\left[N_{\mathrm{H}_{2}}^{1 / 2} N_{\mathrm{CH}_{4}}\left(\frac{h^{2}}{2 \pi m_{\mathrm{H}_{2}} T}\right)^{3 / 4}\left(\frac{m_{\mathrm{CH}_{3}}}{m_{\mathrm{CH}_{4}}}\right)^{3 / 2} V^{\prime}\right]^{5} .
\end{gathered}
$$

Each of the five summands of the sum in Eq. (14) has the form

$$
\theta C_{5}^{p}\left(-\frac{E_{1}}{T^{3}} h \dot{T} \frac{\Omega_{1}}{\Omega_{\mathrm{btn}}}\right)^{p} \exp \left(-f_{p}\right)(t-\tau)^{3},
$$

where

$$
f_{p}=\left[\left(\Delta G_{\mathrm{c}}+5 E_{1}\right)-p\left(E_{\mathrm{btn}}^{(\mathrm{diss})}-E_{1}\right)\right] / T+\varsigma
$$

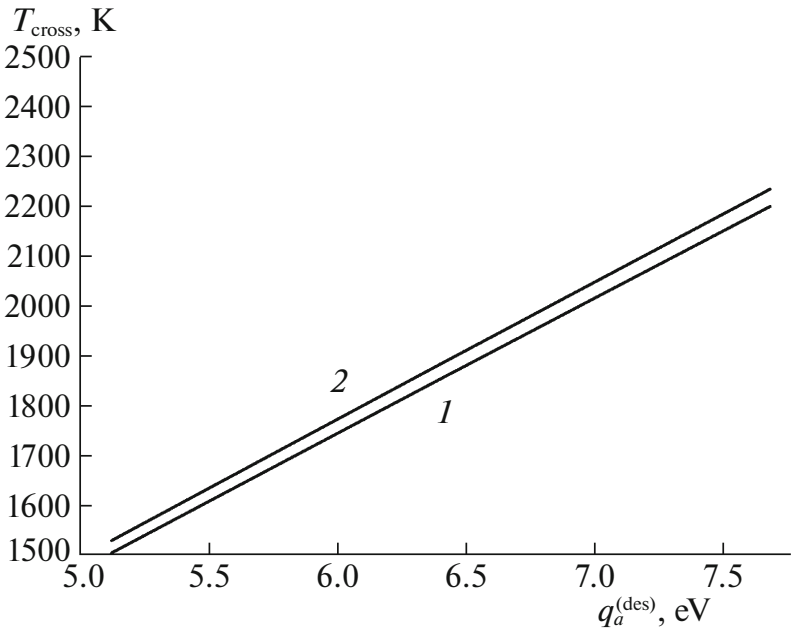

Fig. 4. Temperature of nucleation versus the activation energy of desorption from the surface of a diamond cluster as a varied parameter at temperature growth rates $\dot{T}$ of (1) 800 and (2) $1300 \mathrm{~K} / \mathrm{s}$.

is sharply changing function $\varsigma$. Since the summands have the same structure, we shall give the result from solving Eq. (11) by considering only one of them at $p=$ 0 . The maximum rate of the drop in the oversaturation logarithm after the maximum of nucleation is

$$
\left(\frac{d \varsigma}{d T}\right)_{\mathrm{expl}}=-(\dot{T})^{-1} \sqrt{\frac{8}{g_{\mathrm{c}}^{(m)}}\left(\frac{\theta}{2 \Delta N_{1}}\right)^{1 / 2}} \exp \left(-f_{0}^{(m)} / 2\right),
$$

where $f_{0}^{(m)}=\varsigma_{m}+\Delta G_{\mathrm{c}}^{(m)} / T_{m}, \Delta G_{\mathrm{c}}^{(m)}=\sigma_{1} \bar{\varpi}\left(x_{c}^{(m)}\right)^{4} / 4$, and sub/superscript $m$ corresponds to the critical parameters at the moment of maximum oversaturation. The result for Eq. (17) is given in Appendix B.

We can see from a comparison of typical curves $(d \zeta / d T)_{\text {nat }}$ and $(d \varsigma / d T)_{\text {expl }}$ (Fig. 3) that condition $\left|(d \varsigma / d T)_{\text {nat }}\right| \geq\left|(d \varsigma / d T)_{\text {expl }}\right|$ is met for both graphite and diamond in a narrow range of temperatures of no more than several tens of Kelvins. The temperature at the point of intersection between curves $\left|(d \varsigma / d T)_{\text {nat }}\right|$ and $\left|(d \zeta / d T)_{\text {expl }}\right|$ in this case depends weakly on derivative $\dot{T}$. The curves (or virtually straight lines) 1 and 2 in Fig. 4 correspond to different values of $\dot{T}=800$ and $1300 \mathrm{~K} / \mathrm{s}$. However, we should note that the model was originally designed for high values of $\dot{T}$, and the result cannot be extrapolated to $\dot{T} \rightarrow 0$.

In contrast, Fig. 4 also shows the moment of nucleation depends strongly on $q_{\mathrm{a}}^{(\mathrm{des})}$. It is natural to assume it behaves like $q_{\mathrm{btn}}^{(\mathrm{des})}$, which (as we found in [1]) differs in the channels of diamond and graphite within the spread of initial clusters and the way in which they are 


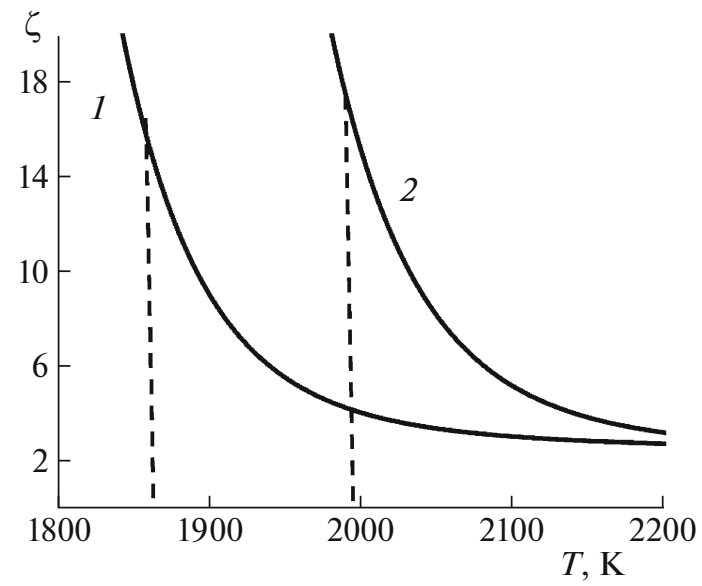

Fig. 5. Changes in $\zeta=\ln S$ in the channels of (1) graphite and (2) diamond with rising temperature (the moment of nucleation for both channels is marked with a dashed line). The onset of nucleation for diamond clusters corresponds to slightly higher oversaturation.

calculated. Let us assume, however, that $q_{\mathrm{a}}^{(\mathrm{des})}$ is exactly equal to $q_{\mathrm{btn}}^{(\mathrm{des})}$ and is slightly higher for diamond than for graphite: $q_{\mathrm{a}}^{\text {(des,diam) }}=6.4 \quad \mathrm{eV}$ and $q_{\mathrm{a}}^{\text {(des,graph) }}=6.0 \mathrm{eV}$. The conditions of nucleation in the graphite channel are then reached at a slightly lower temperature (i.e., at an earlier time) and the nucleation of graphite clusters can be expected. However, the requirement of non-oxidation for small clusters establishes a reciprocal relationship between the conditions for the synthesis of graphite and diamond clusters. It can be allowed for by estimating the rate of increase in the number of particles in a cluster (at least at the drop model level) using the equation

$$
\begin{gathered}
d g / d t \\
\sim\left(N_{1} V_{T}^{(1)} \sigma_{\mathrm{crs}}^{(1)}-N_{\text {ox }} V_{T}^{(\text {ox })} \sigma_{\mathrm{crs}}^{(\mathrm{ox})} \exp \left(-E_{\mathrm{ox}} / T\right)\right) g^{2 / 3},
\end{gathered}
$$

where $N_{\text {ox }}$ is the concentration of oxidizer; $v_{T}^{(1)}, \sigma_{\mathrm{crs}}^{(1)}$, $v_{T}^{(\mathrm{ox})}, \sigma_{\mathrm{crs}}^{(\mathrm{ox})}$ are the thermal velocities and gasokinetic cross sections of collisions between a cluster and a fragment or an oxidizer, respectively; $E_{\text {ox }}$ is the activation barrier in the carbon cluster oxidation reaction accompanied by the emergence of a fragment like $\mathrm{CO}_{x}$, and the first summand contains concentration $N_{1}$ of the source (i.e., it is proportional to $\left.\exp (\zeta)\right)$ ). The carbon cluster will grow if the right side of Eq. (18) is positive. For this to occur, we must meet the condition

$$
\varsigma \geq \ln \left(N_{\text {ox }} / N_{\mathrm{CH}_{4}}\right)-\left(E_{\text {ox }}-E_{\text {btn }}^{\text {(dis) }}-E_{1}\right) / T+\text { const. }
$$

This condition is satisfied by a very short interval of a change in temperature (the solid intercept in curve 1) in the graphite curve of Fig. 3, and a much longer interval in the diamond curve (curve 2). The difference between the effective energies of oxidation
$E_{\mathrm{ox}}^{(\mathrm{diam})}-E_{\mathrm{ox}}^{(\mathrm{graph})}$ of the diamond and graphite channels is thus higher than the one for the desorption bottlenecks of small hydrocarbon fragments: $q_{\mathrm{a}}^{\text {(des,diam) }}-q_{\mathrm{a}}^{\text {(des,graph) }}[1]$. Energy $E_{\mathrm{ox}}^{\text {(graph) }}$ of the oxidation of graphite clusters in Eq. (18) is much lower, and their emergence requires much greater oversaturation. The energy of oxidation is much lower when diamond nuclei emerge. The admissible oversaturation can also be lower but is actually higher than in the graphite channel (Fig. 5), and the diamond clusters that emerge are viable.

The formation of diamond nuclei is therefore more advantageous than that of graphite nuclei for the synthesis of carbon clusters upon a sharp increase in temperature. As was noted above, the model shows that temperature $T_{\text {cross }}$ of nucleation grows along with $\dot{T}$ (Fig. 4). As $\dot{T}$ grows, the concentrations of diamond nuclei $N_{\bar{g}}^{(\text {diam) }}$ and contrast $N_{\bar{g}}^{(\text {diam) }} / N_{\bar{g}}^{\text {(graph) }}$ (i.e., the ratio between the concentration of clusters with a certain average size $\bar{g}$ ) are also reduced. Both parameters were determined at the same level of estimation as in Eq. (18):

$$
\begin{aligned}
& d N_{\bar{g}} / d t=a S^{2} \exp \left(-2 E_{1} / T\right) \\
& -b N_{\mathrm{Ox}} N_{\bar{g}} \exp \left(-E_{\mathrm{ox}}^{(\text {diam })} / T\right),
\end{aligned}
$$

where $a$ and $b$ are the universal constants (but naturally different) for graphite and diamond. At the terminal stage of nucleation, where the left side of Eq. (19) becomes zero, the concentration of diamond nuclei

$$
N_{\bar{g}} \sim \exp \left(\left(E_{\text {ox }}^{\text {(diam) }}-2 E_{1}\right) / T\right)
$$

then falls as the temperature rises, as does the ratio

$$
\begin{gathered}
N_{\bar{g}}^{\text {(diam) }} / N_{\bar{g}}^{\text {(graph) }} \sim\left(S_{\text {diam }}^{2} / S_{\text {graph }}^{2}\right) \\
\times \exp \left(\left(E_{\text {ox }}^{\text {(diam) }}-E_{\text {ox }}^{\text {(graph) }}\right) / T\right), \\
E_{\text {ox }}^{\text {(diam) }}>E_{\text {ox }}^{\text {(graph) }} .
\end{gathered}
$$

The number of atoms $g$ in a diamond cluster also falls as $T$ rises, due to condition (19). The clusters grow more slowly and are more quickly oxidized.

After the nucleation of clusters within a temperature range of several tens of Kelvins, they can be conserved only there is a transition from a rise to a drop in temperature toward the substrate. Such transitions do occur in both HFCVD and MWCVD. In MWCVD, it happens because the node of electrical field oscillations at which the field concentration is greatest is always at a certain distance from the substrate. The temperature estimated for carbon clusters as 1900$2000 \mathrm{~K}$ in a self-consistent solution must be compared to substrate temperature $T_{\mathrm{s}}$ and gas temperature $T_{\mathrm{g}}$ that are typical for CVD. Compared to known values of $T_{\mathrm{s}}$ and $T_{\mathrm{g}}$, it is evidently overestimated. However, it corresponds perfectly to the hot filament temperature in HFCVD. In the typical conditions of microwave 
assisted CVD diamond synthesis, which is done in a fast gas flow, there is not enough time for temperatures $T_{\mathrm{g}}$ of the heavy component, $T_{\mathrm{e}}$ of electrons, and $T_{\mathrm{v}}$ of vibrations in carbon clusters to reach identical values. At the same time, different components of the synthesis of carbon nuclei are sensitive to different temperatures.

For small clusters and hydrogen in the equilibrium distributions and probabilities of reactions, effective temperature $T_{\text {eff }}$ can be estimated as the geometric mean of temperatures $T_{\mathrm{g}}$ and $T_{\mathrm{e}}$. Using the preexponent of forward $\mathrm{H}_{2}$ dissociation in results from numerical calculations for isothermic hydrogen plasma $[8,9]$ and knowing the independent ratio between $T_{\mathrm{g}}$ and $T_{\mathrm{e}}$, we can find these parameters separately and compare $T_{\mathrm{g}}$ to the known temperatures of diamond synthesis. To estimate the ratio between $T_{\mathrm{g}}$ and $T_{\mathrm{e}}$, we can use the results from calculations for pure hydrogen plasma and the modeling of MWCVD in the geometry of diamond synthesis $[10,11]$.

Distribution $T_{\mathrm{e}}(\mathbf{r})$ from [11] is shown in the inset in Fig. 6, which restores dependence $T_{\mathrm{e}}(T)$ from distributions $T(\mathbf{r})$ and $T_{\mathrm{e}}(\mathbf{r})$. Although the calculations in [11] were done at a certain plasma-generating microwave power, the flow rate, the geometry of electrodes, and the dynamics of the change in relationship $\left(T_{\mathrm{e}}-\right.$ $T$ ) were assumed to be universal. The gas temperature then lay below $1000 \mathrm{~K}$ at an effective temperature of nucleation of $\sim 2000 \mathrm{~K}$. This value is slightly higher than the substrate temperature typical of diamond synthesis, as it should be. Estimates below show the above $T_{\text {eff }}$ corresponds to the ratio between $T_{\mathrm{g}}$ and $T_{\text {eff }}$ also when $T_{\text {eff }}$ is understood to be characteristic vibrational temperature $T_{\mathrm{v}}$ of clusters The distinctions between $T_{\mathrm{v}}$ and $T_{\mathrm{g}}$ are due to the non-isothermic effects of nucleation in small clusters: $T_{\mathrm{v}}$ is higher than $T_{\mathrm{g}}$ because of the adsorption of fragments from an oversaturated vapor and liberation of the heat of desorption. The temperatures are in this case equal because of nonelastic collisions between clusters and hydrogen molecules. Rate $(d / d t)\left(T_{\mathrm{v}}-T_{\mathrm{g}}\right)$ of the change in the difference between temperatures $T_{\mathrm{g}}$ and $T_{\mathrm{v}}$ can then be estimated using the relation

$$
\begin{gathered}
3 g\left(T_{\mathrm{v}}-T_{\mathrm{g}}\right)_{t}^{\prime}=(1 / 4) A_{\mathrm{g}} V_{T}^{\mathrm{CH}_{3}}\left(N_{1} q_{1}\left(1-S^{-1}\right)\right. \\
\left.-N_{\mathrm{H}_{2}} \sqrt{m_{\mathrm{H}_{2}} / m_{\mathrm{CH}_{3}}}\left(2 m_{\mathrm{H}_{2}} / m_{\mathrm{g}}\right)\left(T_{\mathrm{v}}-T_{\mathrm{g}}\right)\right),
\end{gathered}
$$

where $v_{T}^{\left(\mathrm{CH}_{3}\right)}$ is the thermal velocity of methyl radicals; $A_{\mathrm{g}}$ is the surface area of a cluster, estimated as half product of the number of cluster surface atoms $g_{0}(g)$ and the surface areas of non-planar hexagonal cell $A_{1}$ with two carbon atoms. Setting the logarithmic derivatives of $T_{\mathrm{v}}-T_{\mathrm{g}}$ and $T_{\mathrm{g}}$ equal at the level of estimation, we obtain

$$
\begin{gathered}
\left(T_{\mathrm{v}}-T_{\mathrm{g}}\right) / q_{1} \approx\left(\left(A_{\mathrm{g}} / 4\right) V_{T}^{\left(\mathrm{CH}_{3}\right)} N_{1}\right)(3 g(\dot{T} / T) \\
\left.+\frac{1}{4} A_{\mathrm{g}}\left(m_{\mathrm{H}_{2}} / m_{\mathrm{CH}_{3}}\right)^{3 / 2} V_{T}^{\left(\mathrm{CH}_{3}\right)} N_{\mathrm{H}_{2}}\left(2 m_{\mathrm{CH}_{3}} / m_{\mathrm{g}}\right)\right)^{-1} .
\end{gathered}
$$

In different regimes, $T_{\mathrm{g}}$ proves to be $1.5-2$ times lower than $T_{\mathrm{v}}$.

\section{CONCLUSIONS}

Applied to the typical conditions of effective CVD (PECVD or HFCVD) synthesis of diamonds (i.e., a sharp increase in the temperature near the substrate surface), the criterion of fast heating is true for conditions of the preferable nucleation of carbon clusters via the path of diamond instead of graphite. This criterion is a sharp increase in temperature over time (or its growth with a coordinate under the conditions of a fast gas flow). The absolute value of $T$ is then nearly $1000 \mathrm{~K}$, which is much lower than the typical temperatures of graphitization.

The reason for fast heating is that the concentration of the sources of nucleation (small fragments that make up the material of carbon nuclei from a methane-carbon-oxygen mixture) grows along with temperature almost with no inertia, while the concentration of the same fragments as a saturated vapor over the surface of a growing cluster increases much more slowly.

The synthesis of carbon clusters of both the graphite and diamond types thus proceeds under the conditions of initially giant and then quickly falling oversaturation. The vapor of small carbon fragments stimulating condensation is then more oversaturated in the channel of diamond than in that of graphene (or graphite). With an oxidizer, the rate of oxidation of diamond nuclei is much lower than that of graphite nuclei. Both these results are due to the oxidation and desorption of carbon fragments from the surfaces of diamond clusters being accompanied by the disruption of a great number of carbon bonds.

Our study shows the diamond nuclei formed in the gas phase precipitate onto the substrate in a ready form, without the participation of the substrate itself. However, the criterion of fast heating plays an important role in the technology of CVD diamond synthesis on any substrate. Since the technology of the CDV synthesis of high-quality massive diamonds has basically reached its limits, we can apply this criterion to other ways of synthesizing diamonds. The idea of unsteady temperature dynamics and the possibility of using it to control the channels of gas-phase conden- 


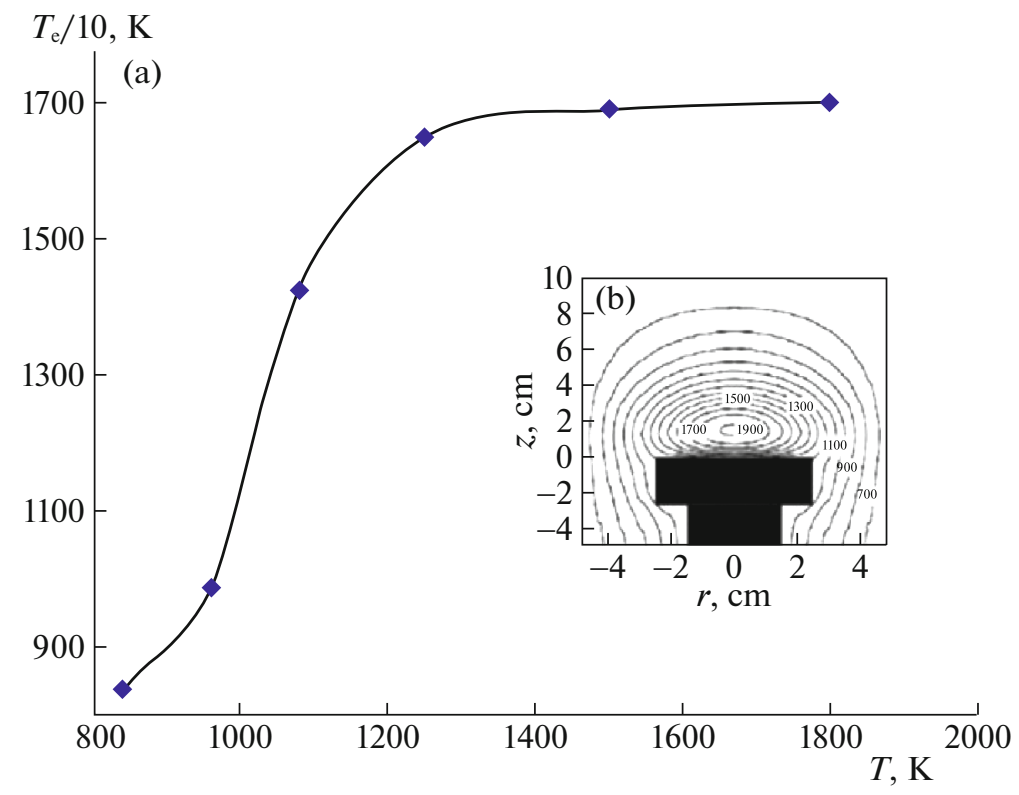

Fig. 6. Electron temperature versus gas temperature in a gaseous hydrogen plasma flow around a substrate, according to the calculated data in [11].

sation could work under the conditions of such synthesis-not on the surface of a substrate, but in the volume of a medium that precludes the free growth of diamond. Such a problem was considered in the last part of our study [12].

\section{APPENDIX A}

\section{Rate of the Multistage Desorption of Small Fragments from the Surface of a Cluster}

The equation for relative concentration $y_{s}$ (with respect to equilibrium) of an intermediate product of the reaction at the sth stage can be written in the diffusion approximation as

$$
\frac{\partial}{\partial t}\left(N_{s}^{(0)} y\right)=\frac{\partial}{\partial \mathrm{s}}\left(w_{s} N_{s}^{(0)} \frac{\partial y}{\partial \mathrm{s}}\right)
$$

where $w_{s}$ is the probability of a forward reaction at the sth stage, and $N_{s}^{(0)}$ is the equilibrium concentration of clusters at the same stage. Since $\partial \ln N_{s}^{(0)} / \partial t=\left(q_{s} / T^{2}\right) \dot{T}$, $\partial \ln \left(w_{s} N_{s}^{(0)}\right) / \partial s=-\left(\partial\left(q_{s}+q_{a, s}\right) / \partial s\right) / T$ (where $q_{s}$ is the overall energy of product dissociation at the sth stage with respect to product 0 ), and using Zeldovich's solution in [8] (i.e., the solution to Eq. (A1) without the left part) for estimates, the ratio between the orders of $y$ and its derivatives at the bottleneck of multistage reaction $s_{\mathrm{btn}}$ is

$$
y\left(s_{\mathrm{btn}}\right)=\left(\int_{s_{\mathrm{btn}}}^{\infty} d s\left(w N_{s}^{(0)}\right)^{-1}\right)\left(\int_{0}^{\infty} d s\left(w N_{s}^{(0)}\right)^{-1}\right)^{-1} \sim \frac{1}{2},
$$

$$
\partial y / \partial s \sim 1 / 2 s_{\mathrm{btn}}, \quad \partial^{2} y / \partial s^{2} \sim 1 / 2 s_{\mathrm{btn}}^{2} .
$$

Since $s_{\mathrm{btn}}$ is high, the term with the first derivative on the right side predominates: $\left(\partial \ln \left(w_{s} N_{s}^{(0)}\right) / \partial s\right)(\partial y / \partial s) \gg$ $\partial^{2} y / \partial s^{2}$. Equation (A1) is converted into the first-order equation in variables $(t, s)$ at $q_{s}>T$ :

$$
\begin{gathered}
\dot{y}+\frac{q_{s}^{(\mathrm{des})}}{T^{2}} \dot{T} y=\frac{(d / d s)\left(q_{s}^{(\mathrm{des})}+q_{a}^{(\mathrm{des})}(s)\right)}{h} \\
\times \exp \left(-\frac{q_{a}^{(\mathrm{des})}(s)}{T}\right) \frac{\partial y}{\partial s}
\end{gathered}
$$

where energies $q$ are written with superscript (des) to indicate they describe desorption.

Assuming that derivative $\dot{y}$ on the left side of Eq. (A2) can be ignored as in Eq. (2), we obtain an ordinary differential equation expressed through $s$ and at $q_{s}^{\text {(des) }}>T$ having the solution

$$
\begin{gathered}
y(s) \approx y(s=0) \\
\times \exp \left(-\frac{h \dot{T}}{T^{2}} \int_{0}^{s} \frac{q_{\mathrm{s}^{\prime}}^{(\mathrm{des})}}{\left(d / d s^{\prime}\right)\left(q_{s^{\prime}}^{(\mathrm{des})}+q_{a, s^{\prime}}^{(\mathrm{des})}\right)} \exp \left(\frac{q_{a, s^{\prime}}^{(\mathrm{des})}}{T}\right) d s^{\prime}\right)
\end{gathered}
$$

at the bottleneck, we must specify $s=s_{\mathrm{btn}}$. 
The possibility of ignoring derivative $\dot{y}$ is due to the condition

$$
\begin{gathered}
\dot{y} /\left(\left(q_{s}^{\text {(des) }} / T^{2}\right) \dot{T} y\right) \approx\left(h \dot{T} q_{a}^{(\mathrm{des})} \mathrm{s}_{\mathrm{btn}}^{2} / 2 q_{s}^{(\mathrm{des})} T^{2}\right) \\
\times \exp \left(q_{a}^{\text {(des) }} / T\right) \ll 1 .
\end{gathered}
$$

At an activation energy of $5 \mathrm{eV}$, this condition is met at temperatures above $0.15 \mathrm{eV}$, which lie the region of the nucleation of carbon clusters. Under the condition opposite to Eq. (A4), the solution has the form of a random function of the argument

$$
s-\left[\left(d q_{s}^{(\mathrm{des})} / d s\right) T^{2} / h \dot{T} q_{a, s}^{(\mathrm{des})}\right] \exp \left(-q_{a}^{(\mathrm{des})} / T\right),
$$

where $y(\phi)=\exp \left(-\phi^{2}\right)$ falls at the initial moment by $e^{2}$ times in the interval of $s$ changing from 0 to 1 . A dependence of this kind can be easily constructed using Eq. (A3) (e.g., in the interval of $\left(h \dot{T} q_{a}^{(\mathrm{des})} s_{\mathrm{btn}}^{2} / 2 q_{s}^{(\mathrm{des})} T^{2}\right) \exp \left(q_{a}^{(\mathrm{des})} / T\right)$ from $1 / 2$ to 2 , according to the value and derivative).

\section{APPENDIX B}

As in the classic problem of explosive nucleation, the integral on the right side of Eq. (11) is selected according to the saddle point. The exponent in function $f$ is expanded as a series in powers $\left(\zeta_{m}-\zeta\right)$ to a quadratic term, and the difference itself is expanded as a series in difference powers $t_{m}-t$, where $t_{m}$ is the moment of reaching maximum $\zeta=\zeta_{m}$. This approach is feasible if the exponent of the function "minus $f$ " changes sharply with respect to $\zeta$ itself in the vicinity of the maximum (Fig. 7). At variable temperatures, we must meet another requirement: the temperature must change more smoothly than $\zeta$. Then $\exp \left(-f_{0}(\tau)\right)=\exp \left(-f_{0}^{(m)}\right) \exp \left(\left(\alpha / 2-\sigma_{1} \varpi\left(x_{\mathrm{c}}^{4}\right)_{t t}(t=\right.\right.$ $\left.\left.\left.t_{m}\right) / 6 T^{(m)}\right)\left(\tau-t_{m}\right)^{2}\right)$, where $\alpha=\left|\zeta_{t t}^{\prime \prime}\right|\left(t=t_{m}\right)$.

With the above definitions, the exponent of the subintegral expression in Eq. (14) has the Gaussian form

$$
\begin{aligned}
\exp \left(-f_{0}\right) & =\exp \left(-f_{0}^{(m)}\right) \exp \left[-\gamma\left(t_{m}-\tau\right)^{2}\right], \\
\gamma & =(\alpha / 2)\left(g_{\mathrm{c}}^{(m)} / 2-1\right),
\end{aligned}
$$

and

$$
J(\tau) g\left(\tau, t_{m}\right)=\theta \exp \left(-f_{0}^{(m)}\right) \exp \left[-\gamma\left(t_{m}-\tau\right)^{2}\right]\left(t_{m}-\tau\right)^{3} .
$$

At the moment of maximum $t_{m}$, integral $\int_{0}^{t_{m}} J(\tau) g(\tau, t) d \tau \approx \theta \exp \left(-f_{0}^{(m)}\right) \int_{0}^{\infty} \exp \left[-\gamma \tau^{2}\right] \tau^{3} d \tau$. At the final moment of the explosive nucleation stage (when $\Delta N_{1}=N_{1} / 2$ ), Eq. (11) takes the form

$$
\Delta N_{1}=\theta \exp \left(-f_{0}^{(m)}\right) / \gamma^{2} .
$$

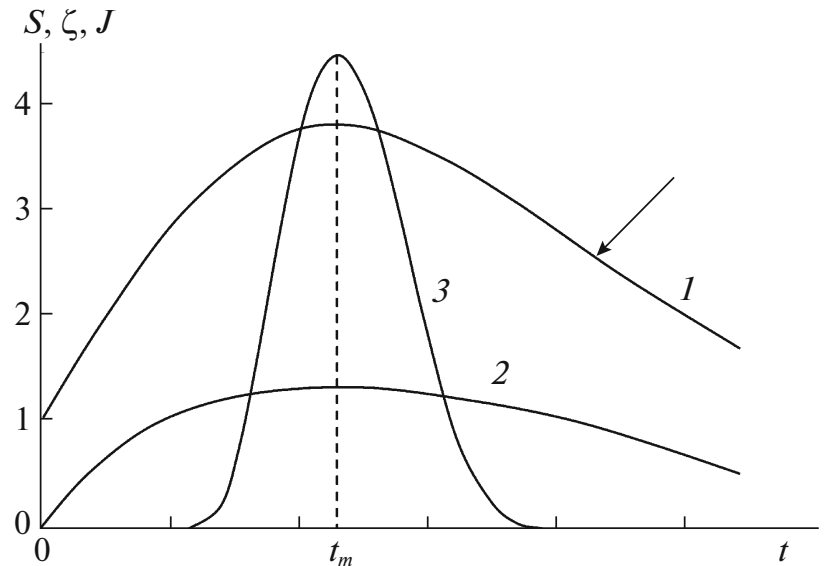

Fig. 7. Hierarchy of characteristic times underlying the traditional explosive nucleation model $[8,9]$ : $(1,2)$ are oversaturation $S$ and $\zeta=\ln S ; J$ is the density of the flux of generated clusters through the bottleneck in the space of sizes. The scale of flux values is nominal. The moment when the rate of the drop in oversaturation is greatest is shown with an arrow.

In light of all the exponents, this result is replaced by

$$
\begin{aligned}
\Delta N_{1}=\left(\theta / \gamma^{2}\right) \sum_{p=0}^{5} & C_{5}^{p}\left(-\left(E_{1} h \dot{T} / T^{3}\right)\left(\Omega_{1} / \Omega_{\mathrm{btn}}\right)\right)^{p} \\
& \times \exp \left(-f_{p}^{(m)}\right),
\end{aligned}
$$

where

$$
f_{p}^{(m)}=\left[\left(\Delta G_{\mathrm{c}}^{(m)}+5 E_{1}\right)-p\left(E_{\mathrm{btn}}^{(\mathrm{diss})}-E_{1}\right)\right] / T+\varsigma_{m} .
$$

Using Eq. (A4), the oversaturation curve half width incorporated into multiplier $\gamma$ and rate of descent $\zeta$ after the nucleation maximum is found from the known $\Delta N_{1}$ and $f_{m}$.

\section{OPEN ACCESS}

This article is licensed under a Creative Commons Attribution 4.0 International License, which permits use, sharing, adaptation, distribution and reproduction in any medium or format, as long as you give appropriate credit to the original author(s) and the source, provide a link to the Creative Commons license, and indicate if changes were made. The images or other third party material in this article are included in the article's Creative Commons license, unless indicated otherwise in a credit line to the material. If material is not included in the article's Creative Commons license and your intended use is not permitted by statutory regulation or exceeds the permitted use, you will need to obtain permission directly from the copyright holder. To view a copy of this license, visit http://creativecommons.org/licenses/by/4.0/. 


\section{REFERENCES}

1. N. I. Alekseev and V. S. Khadutin, Russ. J. Phys. Chem. A (2021, in press).

2. S. V. Digonskii and V. V. Ten, Unknown Hydrogen (the Role of Hydrogen in the Polymorphism of Solids, the Processes of Solid-Phase Reduction of Oxides and Sintering of Powders) (Nauka, St. Petersburg, 2006) [in Russian].

3. H. Eyring, S. H. Lin, and S. M. Lin, Basic Chemical Kinetics (Wiley, New York, 1980).

4. S. A. Kukushkin and A. V. Osipov, Phys. Usp. 41, 983 (1998).

5. Yu. P. Raizer, Sov. Phys. JETP 10, 1229 (1959).

6. D. I. Zhukhovitskii, A. G. Khrapak, and I. T. Yakubov, Teplofiz. Vys. Temp., No. 5, 982 (1983).
7. D. I. Zhukhovitskii, A. G. Khrapak, and I. T. Yakubov, Teplofiz. Vys. Temp., No. 6, 1197 (1983).

8. Ya. B. Zel'dovich, Zh. Eksp. Teor. Fiz. 12, 525 (1942).

9. L. M. Biberman, V. S. Vorob'ev, and I. T. Yakubov, $K i-$ netics of Nonequilibrium Low-Temperature Plasma (Nauka, Moscow, 1982) [in Russian].

10. J. E. Butler, Y. A. Mankelevich, A. Cheeseman, et al., J. Phys.: Condens. Matter, No. 21, 364201 (2009).

11. K. Hassouni, G. Lombardi, X. Duten, et al., Plasma Sources Sci. Technol., No. 15, 117 (2006).

12. N. I. Alekseev, V. S. Khadutin, and I. K. Khmel'nitskii, Russ. J. Phys. Chem. A (2021, in press).

Translated by E. Glushachenkova 